\title{
Stress relaxation of narrow molar mass distribution polystyrene following uniaxial extension
}

Nielsen, Jens Kromann; Rasmussen, Henrik K.; Hassager, Ole

Published in:

Journal of Rheology

Link to article, DOI:

$10.1122 / 1.2930872$

Publication date:

2008

Document Version

Publisher's PDF, also known as Version of record

Link back to DTU Orbit

Citation (APA):

Nielsen, J. K., Rasmussen, H. K., \& Hassager, O. (2008). Stress relaxation of narrow molar mass distribution polystyrene following uniaxial extension. Journal of Rheology, 52(4), 885-899. https://doi.org/10.1122/1.2930872

\section{General rights}

Copyright and moral rights for the publications made accessible in the public portal are retained by the authors and/or other copyright owners and it is a condition of accessing publications that users recognise and abide by the legal requirements associated with these rights.

- Users may download and print one copy of any publication from the public portal for the purpose of private study or research.

- You may not further distribute the material or use it for any profit-making activity or commercial gain

- You may freely distribute the URL identifying the publication in the public portal 


\title{
Stress relaxation of narrow molar mass distribution polystyrene following uniaxial extension
}

\author{
Jens Kromann Nielsen ${ }^{\text {a) }}$ \\ The Danish Polymer Center, Department of Chemical and Biochemical \\ Engineering, Technical University of Denmark, DK-2800 Kgs. Lyngby, Denmark \\ Henrik Koblitz Rasmussen \\ The Danish Polymer Center, Department of Mechanical Engineering, \\ Technical University of Denmark, DK-2800 Kgs. Lyngby, Denmark \\ Ole Hassager \\ The Danish Polymer Center, Department of Chemical and Biochemical \\ Engineering, Technical University of Denmark, DK-2800 Kgs. Lyngby, Denmark
}

(Received 29 November 2006; final revision received 12 March 2008)

\begin{abstract}
Synopsis
The stress in the startup of uniaxial elongational flow until steady state, followed by stress relaxation, has been measured for a narrow molar mass distribution polystyrene melt with a molecular weight of $145 \mathrm{~kg} / \mathrm{mol}$. The experiments are conducted on a filament stretching rheometer, where a closed loop control of the midfilament diameter ensures controlled uniaxial extension. The closed loop control algorithm is extended to apply to the stress relaxation part of the experiment. It ensures a constant midfilament diameter, by controlling the motion of the end plates. By dividing the measured stress with the theoretically predicted stress from the Doi and Edwards model during relaxation, the stretch factors corresponding to each imposed stretch rate are obtained. These stretch factors converge to a unique envelope and eventually converge to unity for long times for all measured elongational rates. (c) 2008 The Society of Rheology.
\end{abstract}

[DOI: $10.1122 / 1.2930872]$

\section{INTRODUCTION}

The progress in the understanding of the nonlinear properties of polymer melts in elongational flow seems to be limited by the scarcity of data for well-characterized narrow molar mass distribution (NMMD) polymer melts. Bach et al. (2003a), Luap et al. (2005), Nielsen et al. (2006a), and Rasmussen et al. (2008) have presented transient and steady elongational viscosity of NMMD linear polystyrene melts. Nielsen et al. (2006b) have presented data on branched melts in the form of a multiarm $A_{n}-C-C-A_{n}$ pom-pom polystyrene and an $A_{n}-C$ asymmetric star polystyrene where $n$ is the average number of arms.

\footnotetext{
a) Author to whom correspondence should be addressed; electronic mail: kromann_@hotmail.com
} 
None of the repetation-based models developed prior to the publishing of these data were effectively able to predict the extensional flow behavior of these polymers melts. They include the pom-pom model [McLeish and Larson (1998)] for branched polymers and an immense variety of concepts for linear polymers, such as Doi and Edwards (1979), Marrucci and Grizzuti (1988), Pearson et al. (1989), Mead et al. (1998), Fang et al. (2000), Ianniruberto and Marrucci (2001), and Schieber et al. (2003). Recently Marrucci and Ianniruberto (2004) introduced the idea of "interchain pressure" and Likhtman (2005) creation and destruction of "slip links" into the basic repetation picture for linear melts. The interchain pressure concept agrees well with experimental findings and was adopted by Wagner et al. $(2005,2008)$ within an integral molecular stress function formulation.

Here we will present a new technique for direct measurements of the stress relaxation following steady uniaxial extensional flow in an NMMD linear polystyrene melt in order to gain further insight into the dynamics of polymer melts.

To our knowledge the only published results for highly nonlinear stress relaxation after extension of polymer melts are those of Sentmanat et al. (2005) and Wang et al. (2007). They presented uniaxial elongational startup data, for broadly distributed low density polyethylene and NMMD styrene-butadiene respectively, followed by stress relaxation performed on a new extensional rheometer, the sentmanat extensional rheometer. On this kind of rheometer the polymer sample is placed between two cylindrical drums that are rotated in opposite directions, and thereby stretch the sample generating an elongation flow. The drums are stopped, and the stress is measured in the following relaxation. This technique is limited at high strains by the appearance of a progressive thinning of the sample ending with a ductile necking.

The first controlled filament stretching rheometer (FSR) was developed by Sridhar et al. (2003). A general description of the FSR may be found in the review by McKinley and Sridhar (2002). The FSR has been used to examine the stress relaxation following uniaxial extension on polymer solutions [Spiegelberg and McKinley (1996a); Orr and Sridhar (1996); Nieuwkoop and von Czernicki (1996); Doyle et al. (1998); Bhattacharjee et al. (2003); Shaqfeh et al. (2004)]. In these experiments a cylindrical polymer sample is placed between two cylindrical end plates that are moved apart. The velocity of the moving end plates can be arrested whereby the imposed deformation stops and the fluid relaxes. Applying this method on polymer melts results in a progressive thinning of the sample.

The use of laser microscopy in the FSR has enabled online strain confirmation, and subsequently closed loop control of the filament diameter by actively controlling when the end plate separation is initiated. This was proposed by Anna et al. (1999). In our paper we present a new method of measuring the stress relaxation, after startup of uniaxial elongational flow, that uses a closed loop control of the end plates in the filament stretching rheometer to ensure true cessation of the flow, corresponding to no change in the midfilament diameter during stress relaxation.

\section{MATERIAL}

The polystyrene used in this work has been synthesized by anionic polymerization in an argon atmosphere according to the procedure suggested by Ndoni et al. (1995). The synthesis is performed with cyclohexane as solvent and styrene is initiated by sec-butyl lithium at room temperature. When the styrene has reacted fully we added degassed 


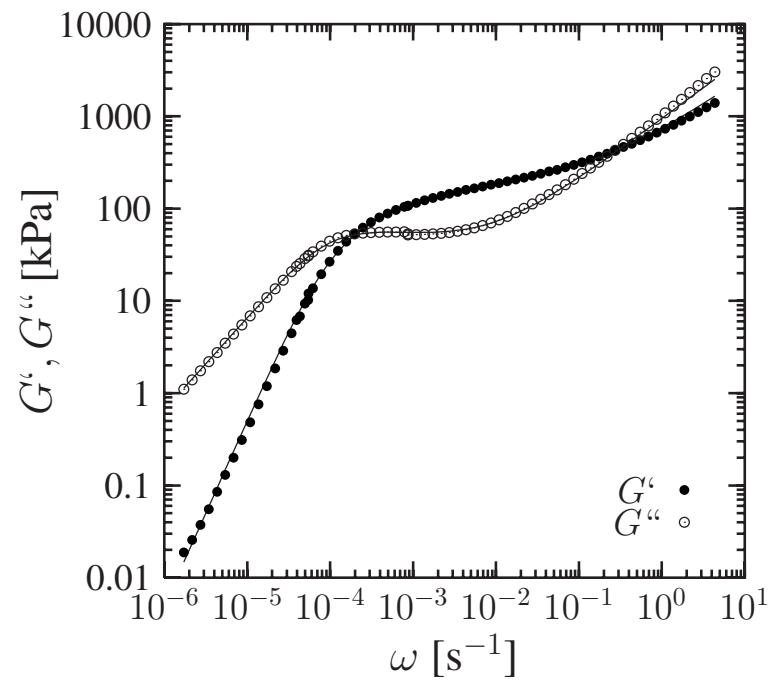

FIG. 1. Measured loss, $G^{\prime \prime}$ (open circles; ${ }^{\circ}$ ) and storage moduli, $G^{\prime}$ (bullets; $\bullet$ ) both as a function of the angular frequency, $\omega$. The data are obtained from small angle oscillatory shear rheometry. The experiments have been performed at 120,130 , and $150{ }^{\circ} \mathrm{C}$, and shifted to a reference temperature of $T_{0}=120^{\circ} \mathrm{C}$. The solid lines $(-)$ are the least-squares fitting to the BSW model in Eq. (2).

methanol to terminate the reaction. The polystyrene was precipitated in iso-propanole, filtered, and vaccum dried according to the protocol described in Schausberger and Schindlauer (1985) before rheological use.

The molar mass was determined by size exclusion chromatography with tetrahydrofuran as eluent and employing a column of a $5 \mu \mathrm{m}$ gaurd column $300 \times 8 \mathrm{~mm}^{2}$ column (PLgel Mixel C and Mixed D). The system is equipped with a differential diffractive index detector. Based on calibrations with narrow molecular weight polystyrene standards, the values of $M_{w}$ and $M_{w} / M_{n}$ were determined to be, respectively, $145 \mathrm{~kg} / \mathrm{mol}$ and 1.03 .

\section{A. Mechanical spectroscopy and linear viscoelasticity}

The linear viscoelastic properties were obtained using small amplitude oscillatory shear measurements on an AR2000 rheometer from TA Instruments. We used a $25 \mathrm{~mm}$ plate-plate geometry and measured the loss and storage moduli, $G^{\prime}$ and $G^{\prime \prime}$, at temperatures ranging from 120 to $150{ }^{\circ} \mathrm{C}$. The data were converted to $120^{\circ} \mathrm{C}$ using timetemperature super positioning with a shift factor $a_{T}=23$ between 120 and $130{ }^{\circ} \mathrm{C}$ and $a_{T}=44$ between 130 and $150{ }^{\circ} \mathrm{C}$. These numbers represent the ratio between frequencies for the crossover of $G^{\prime}$ and $G^{\prime \prime}$ at the respective temperatures.

The measurements for the loss modulus $G^{\prime \prime}$ and the storage modulus $G^{\prime}$ are shown in Fig. 1 as function of the angular frequency $\omega$ in the oscillation. The linear viscoelastic (LVE) data are fitted with a continuous BSW (Baumgaertel-Schausberger-Winter) relaxation spectrum, given by Baumgaertel et al. (1990).

$$
G(t)=\int_{0}^{\infty} \frac{H(\tau)}{\tau} e^{(-t / \tau)} d \tau,
$$


TABLE I. Linear viscoelastic and molecular weight properties of the NMMD polystyrene melts at $120^{\circ} \mathrm{C}$. The $n_{e}, n_{g}$ and $\tau_{c}$ constants in the BSW model are obtained from Jackson and Winter (1995) and $G_{N}^{0}$ $=244 \mathrm{kPa}$ from Bach et al. (2003a). The two parameters $\tau_{W}$ and $\tau_{\mathrm{MI}}$ are the time constants for specific models for the nonlinear relaxation of stretch.

\begin{tabular}{lc}
\hline \hline$M_{w}[\mathrm{~kg} / \mathrm{mol}]$ & $145 \mathrm{~kg} / \mathrm{mol}$ \\
$M_{w} / M_{n}$ & 1.03 \\
$G_{N}^{0}$ & $244 \mathrm{kPa}$ \\
$n_{e}$ & 0.23 \\
$n_{g}$ & 0.67 \\
$\tau_{\max }$ & $14000 \mathrm{~s}$ \\
$\tau_{c}$ & $12 \mathrm{~s}$ \\
$\tau_{a}$ & $7700 \mathrm{~s}$ \\
$\tau_{W}$ & $7000 \mathrm{~s}$ \\
$\tau_{\mathrm{MI}}$ & $5500 \mathrm{~s}$ \\
\hline \hline
\end{tabular}

$$
H(\tau)=n_{e} G_{N}^{0}\left[\left(\frac{\tau}{\tau_{\max }}\right)^{n_{e}}+\left(\frac{\tau}{\tau_{c}}\right)^{-n_{g}}\right] h\left(1-\tau / \tau_{\max }\right),
$$

where $h(x)$ is the Heaviside step function, $n_{e}$ is the slope of the $\left(\log (\omega), \log G^{\prime}\right)$ curve at intermediate frequencies $\omega, n_{g}$ is the slope of $\left(\log (\omega), \log G^{\prime \prime}\right)$ for $\omega \rightarrow \infty$, and $\tau_{c}$ is the crossover relaxation time.

The lines in Fig. 1 are the best fit [Rasmussen et al. (2000)] of the BSW model. The parameters are given in Table I.

We define a characteristic relaxation time:

$$
\tau_{a}=\frac{\int_{0}^{\infty} G(s) s d s}{\int_{0}^{\infty} G(s) d s} \approx \tau_{\max }\left(\frac{1+n_{e}}{2+n_{e}}\right),
$$

which is also given in Table I. We use the relaxation time $\tau_{a}$ to define a Deborah number for the elongational flow, $\mathrm{De}=\dot{\boldsymbol{\epsilon}} \cdot \tau_{a}$, since it is given that $\tau_{a}$ within $5 \%$ is equal to the Doi-Edwards repetation time, as discussed in Bach et al. (2003a).

\section{ELONGATIONAL TECHNIQUE}

The elongational experiments were performed using an FSR surrounded by a thermostated environment as described by Bach et al. (2003b). The polystyrene samples were molded into cylindrically formed pellets with height $\left(L_{i}\right)$ of $2 \mathrm{~mm}$ and diameter of $\left(2 R_{i}\right)$ of $9 \mathrm{~mm}$. The pellet is placed in the rheometer between two parallel steel cylinders having the same diameter as the pellet.

All polystyrene samples are prestretched to a radius of $R_{0}$ (and length $L_{0}$ ) at approximately $150{ }^{\circ} \mathrm{C}$ prior to the elongational experiments. The rate at which we prestretch correspond to a Deborah number much less than unity. After prestretching the temperature inside the rheometer is decreased to $120^{\circ} \mathrm{C}$, at which temperature all the elongational experiments are run, except for one. Before starting the experiments the polystyrene samples are relaxed until all residual forces are gone. This is verified by measuring the vertical force, $F$, on the load cell. 
Our experiments are performed under conditions such that the midfilament plane is also the plane of symmetry of the sample. Gravitational sagging is found to be negligible as the sag time is much longer than the duration of the experiments. Therefore we can monitor this potential region of necking, and the distance between the end plates adjusted online obtaining a predefined stretch rate at the neck. Hencky strain is defined and calculated from the radius at the symmetry plane, $R(t)$, from the equation $\epsilon(t)$ $=-2 \ln (R(t) / R(0))$. Moreover, the closed loop proportional regulator from Bach et al. (2003b) has been extended to control the fixed midfilament diameter during the stress relaxation.

An expression for the difference between the axial and radial stress in the midfilament plane can be obtained from a force balance on the lower half of the filament [Szabo (1997); Szabo and McKinley (2003)]. In the absence of inertial and surface tension effects we write the equation in the form:

$$
\left\langle\sigma_{z z}-\sigma_{r r}\right\rangle+\frac{1}{2}\left\langle\sigma_{r r}-\sigma_{\theta \theta}\right\rangle+\frac{1}{2}\left\langle r \sigma_{r z}\right\rangle^{\prime}=\frac{F(t)-m_{f} g / 2}{\pi R(t)^{2}},
$$

where the angular brackets denote an average over the symmetry plane, $F(t)$ is the axial force, $g$ the gravitational acceleration, and $m_{f}$ the weight of the polymer filament. For $v_{\theta}=0$ it can be shown that the second term on the left side is zero for Newtonian fluids. McKinley and Sridhar (2002) state that this argument may be extended to apply for memory fluids. The third terms on the left side have an effect at small extensions when a significant shearing component may be present in the gap. However, we correct for this effect later by the McKinley-Sridhar reverse squeeze flow correction in Eq. (7). Neglect of the interfacial tension $\gamma$ is justified since ratio of the Laplace pressure, $\gamma / R$, to the measured stress $\sigma_{z z}-\sigma_{r r}$ stays below 0.02 in all presented data.

We therefore retain the following terms in the force balance:

$$
\left\langle\sigma_{z z}-\sigma_{r r}\right\rangle=\frac{F(t)-m_{f} g / 2}{\pi R(t)^{2}} .
$$

Based on the arguments that follow we assume moreover that the stress and strain are uniform in the radial direction in the symmetry plane so the angular brackets in Eq. (5) may be omitted. An estimate of the error in the strain may be obtained by consideration of a cylinder (initially of radius $R_{0}$ and length $L_{0}$ ) deformed into one of the shapes in Fig. 3 with length $L$. The material line at the axis will on average have been stretched by the ratio $\lambda_{\min }=L / L_{0}$ which is clearly less than the average stretch of a material line on the surface. However, we may obtain an upper bound on the stretch of a material line at the surface as $\lambda_{\max }=\left(L+2 R_{0}\right) / L_{0}$. Therefore, the radial variation in stretch ratio will be less than $\left(\lambda_{\max }-\lambda_{\min }\right) / \lambda_{\min }=2\left(R_{0} / L_{0}\right) \exp (-\epsilon)$. For the Hencky strains and initial aspect ratios under consideration this term is of order $5 \%$ during stress relaxation. In practice we expect most of the inhomogeneity in the material stretch to take place near the end plates and not at the symmetry plane so we feel it is justified to neglect the radial variation in stretch.

In the absence of a constitutive equation it is less clear how the inhomogeneity in stress may be estimated. Kolte et al. (1997) performed numerical simulations of filament stretching of a dilute and a semidilute poly(isobutylene) solution, modeled respectively by a multimode Oldroyd-B model and a Papanastasiou-Scriven-Macosco model [Bird et al. (1987)]. Based on these simulations (Figs. 19 and 20) we again estimate the error made by omitting the averaging operation in Eq. (5) to be less than about 5\%. With these 
TABLE II. At $120^{\circ} \mathrm{C}$ Prestretch values.

\begin{tabular}{lllllll}
\hline \hline$\dot{\epsilon}\left[s^{-1}\right]$ & $1 \times 10^{-5}$ & 0.0003 & 0.001 & 0.003 & 0.01 & 0.03 \\
\hline$\epsilon_{\text {pre }}$ & 0.57 & 0.84 & 1.27 & 1.28 & 1.71 & 1.97 \\
$\bar{\eta}_{\text {corr }}^{+}(0) / \bar{\eta}^{+}(0)$ & 0.6914 & 0.81 & 0.92 & 0.92 & 0.97 & 0.98 \\
\hline \hline
\end{tabular}

remarks in mind we assume in this work that stress and strain values in the symmetry plane correspond to values in ideal elongation within experimental accuracy.

In the start-up of elongational flow, the sample is at rest for $t<0$, and is applied a constant elongation rate, $\dot{\epsilon}$, for $t>0$. This is followed by no change in the midfilament radius during the stress relaxation starting at a Hencky strain of $\epsilon_{0}$. Generally, the relative deviation between measurement and expected radius never exceeded $3 \%$. The average strain rate, $\dot{\epsilon}$, is calculated from the measurement of the diameter by fitting an exponential function $R(t)=R_{0} e^{-\dot{\epsilon} t / 2}$.

The viscosities during start-up and stress relaxation are defined as

$$
\bar{\eta}^{+}=\frac{\sigma_{z z}-\sigma_{r r}}{\dot{\epsilon}} \quad \text { and } \quad \bar{\eta}^{-}=\frac{\sigma_{z z}-\sigma_{r r}}{\dot{\epsilon}}
$$

respectively. The viscosity during the stress relaxation is defined using the elongational rate from the preceding flow.

Due to the small aspect ratios used in the stretching experiments, an extra shear contribution adds to the measured elongational force during the start-up of the extension flow. This shear contribution is negligible during the stress relaxation. This reverse squeeze flow originates from the no-slip boundary condition at the steel end plates, and is especially important at small Hencky strains. The effect of the additional shear may be eliminated by a correction factor [Spiegelberg and McKinley (1996b)] to yield for the corrected transient uniaxial elongation viscosity:

$$
\bar{\eta}_{\mathrm{corr}}^{+}=\bar{\eta}^{+}\left(1+\frac{e^{-7\left(\epsilon+\epsilon_{\mathrm{pre}}\right) / 3}}{3 \Lambda_{i}^{2}}\right)^{-1} .
$$

Here $\epsilon_{\mathrm{pre}}=2 \ln \left(R_{i} / R_{0}\right)$ is the Hencky strain of the prestretched configuration while $\Lambda_{i}$ $=L_{i} / R_{i}$ is the aspect ratio computed from the initial sample prior to prestretching, fixed at a value of $\Lambda_{i}=0.444$ in all experiments. This correction is analytically correct for very small strains and aspect ratios for all types of fluids. However, the correction is less accurate at increasing strains where the effect of the correction fortunately vanishes [Kolte et al. (1997)]. The values of $\epsilon_{\text {pre }}$ in the presented data are given in Table II together with the initial value of the viscosity correction [Eq. (7)].

One of the characteristics of elongational flow in a filament stretching apparatus is that the extension is not uniform in the vertical direction of the fluid. Because of the no-slip boundary condition at the end plates, the elongation is highest in the middle of the filament, and smallest close to the end plates. When stopping the motion of the end plates the polymer will start to relax, but due to the uneven stretch and therefore stress along the vertical direction, the different fluid elements will relax differently and not necessarily without flow, as illustrated in the following.

We performed a stretching experiment with a rate of $\dot{\epsilon}=0.03 \mathrm{~s}^{-1}$. After $100 \mathrm{~s}$ at a Hencky strain of $\epsilon_{0}=3$ the motion of the end plates $L(t)$ was stopped. It is seen in Fig. 2 that the filament diameter continues to decrease after $100 \mathrm{~s}$, and finally at about $150 \mathrm{~s}$ the sample ruptures. The value of the initial filament diameter $D_{0}$ and the initial plate sepa- 

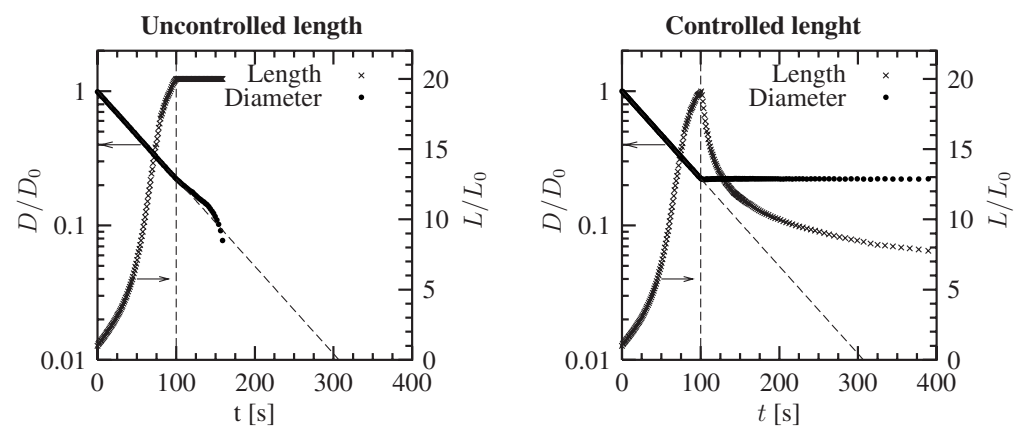

FIG. 2. Evolution of filament diameter, $D(t)$, and plate separation, $L(t)$, for an elongational rate of $\dot{\boldsymbol{\epsilon}}$ $=0.03 \mathrm{~s}^{-1}$ at $120^{\circ} \mathrm{C}$, stretched for $100 \mathrm{~s}$ and then relaxed. During the relaxation, the diameter is kept constant by the closed loop controller in the experiment in the left figure, and the distance between the end plates is kept constant in the experiment in the figure to the right. The initial filament diameter $D_{0}$ and the initial plate separation $L_{0}$ for both the controlled and uncontrolled experiments are: $D_{0}=3.40 \mathrm{~mm}$ and $L_{0}=8.82 \mathrm{~mm}$.

ration $L_{0}$ are given in the label to the figure. The same type of filament thinning for less viscous solutions is seen and utilized in the capillary breakup extensional rheometer to measure the extensional properties of low viscosity liquids [Rodd et al. (2005)]. We, however, wish to avoid this, and therefore the concept of closed loop control of the diameter is used to stop the elongation, simply by moving the end plates back together, as illustrated in Figs. 2 and 3. Figure 3 shows quenched samples. We performed stretching and relaxation experiments with the same elongational rate of $\dot{\epsilon}=0.03 \mathrm{~s}^{-1}$ until a strain of $\epsilon_{0}=3$. This was followed by a quench of the polystyrene samples at several times after cessation of the flow. Note in Fig. 3 that the diameter at the thinnest place, located at the midfilament plane, is the same for all seven filaments.

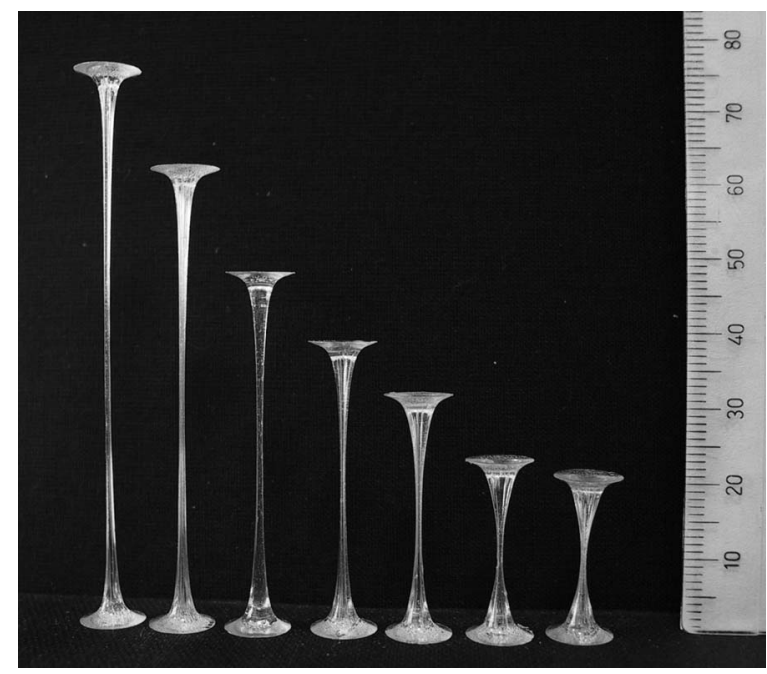

FIG. 3. Quenched polystyrene filaments after cessation of flow. The elongational rate at startup is $\dot{\boldsymbol{\epsilon}}$ $=0.03 \mathrm{~s}^{-1}$, the strain at relaxation is $\epsilon_{0}=3$, and the temperature $120^{\circ} \mathrm{C}$ in all performed experiments. The samples are relaxed, respectively $(0$ s 7 s 47 s 126 s 350 s 3000 s 7000 s), before the quenching. The ruler to the right shows the length in millimeters. 


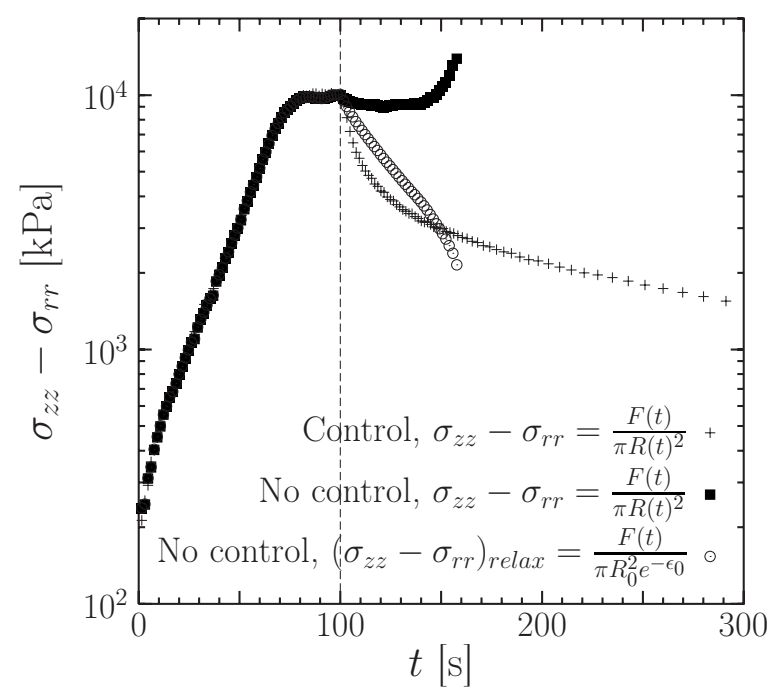

FIG. 4. Measured stress (at $120^{\circ} \mathrm{C}$ ) as a function of time at startup $\left(\dot{\epsilon}=0.03 \mathrm{~s} \mathrm{~s}^{-1}\right)$ and relaxation $\left(\epsilon_{0}=3\right)$ of elongational flow, as in Fig. 2. The diameter is kept constant by the closed loop controller in one of the experiments (+). In the two other curves is the plate separation, $L(t)$, stopped at $t=100 \mathrm{~s}$. The boxes $(\boldsymbol{\square})$ are the calculation of the true stress $\sigma_{r r}-\sigma_{z z}$, where the open circles ( $\left.{ }^{\circ}\right)$ curve are calculated with the assumption of a constant midfilament diameter during the relaxation as $\left(F(t)-m_{f} g / 2\right) /\left(\pi R_{0}^{2} e^{-\epsilon_{0}}\right)$.

The elongational stress for the controlled and uncontrolled stress relaxation is shown in Fig. 4. In both cases we calculate the true stress based on the measured diameter, $\sigma_{z z}-\sigma_{r r}$. The measured stress from the uncontrolled relaxation experiment is almost constant for about $40 \mathrm{~s}$ after stopping the end plates, and finally increases rapidly. The stress in the controlled relaxation experiments decreases immediately after cessation of flow. The importance of having a direct strain verification provided by the laser micrometer is further illustrated in Fig. 4. It includes stress curves calculated with the assumption of a constant midfilament diameter during the relaxation as $\sigma_{z z}-\sigma_{r r}$ $=\left(F(t)-m_{f} g / 2\right) /\left(\pi R_{0}^{2} e^{-\epsilon_{0}}\right)$. It is equal to the true stress in a controlled relaxation experiment but clearly not in an uncontrolled relaxation experiment. The difference between the stresses calculated this way, and the real stress for the uncontrolled experiments is significant.

While the feedback control is crucially needed to follow the stress relaxation after large strain states (as seen in Figs. 2-4) it is less important for relaxation after small strain. To illustrate this we performed an experiment at a rate of $\dot{\epsilon}=0.03 \mathrm{~s}^{-1}$ and stretched to $\epsilon_{0}=0.2$ at $120^{\circ} \mathrm{C}$, as seen in Fig. 5(b) displaying the measured diameter, end plate separation, and stress. The required separation of the plates in this experiment is only about $8 \%$ after cessation of flow. This means that the effect of the closed loop control in this case is not vital. At that small extension the elongational stress is expected to be in good agreement with linear viscoelasticity theory. The elongational viscosity, both at startup and relaxation, is found to be in good agreement with the linear viscoelasticity predictions, as seen in Fig. 5(a).

\section{REPTATION-BASED CONSTITUTIVE MODELS}

It is of interest to compare the experimental observations with predictions from repetation based constitutive theories, since these have monodisperse polymers as the model molecule. 
a)

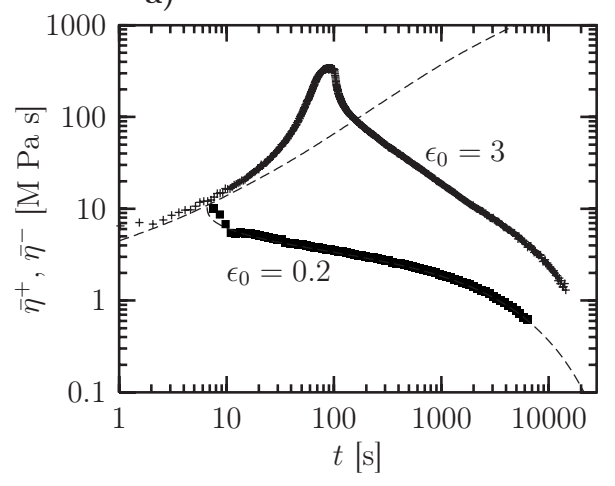

b)

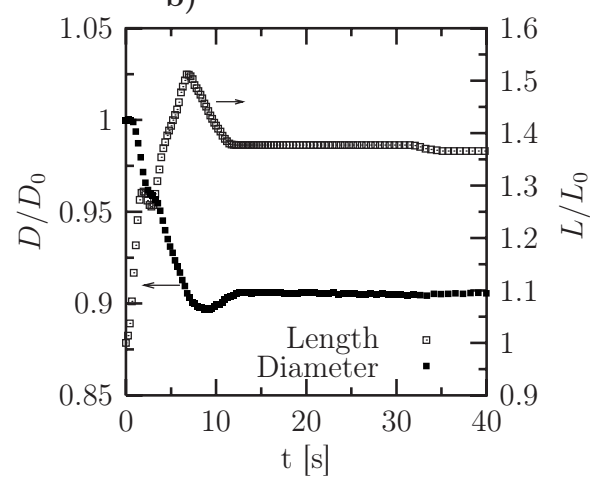

FIG. 5. Figure (a) shows the measured startup and relaxation viscosity performed at an elongational rate of $\dot{\epsilon}=0.03 \mathrm{~s}^{-1}$. In one experiment the filament is stretched for $100 \mathrm{~s}$ and then relaxed (pluses, + ), and in the other experiment the filament is stretched for $6.6 \mathrm{~s}$ and then relaxed, (boxes, $\mathbf{\square})$. The two dotted lines (- - -) are the linear viscoelastic prediction for startup and relaxation after $6.6 \mathrm{~s}$. Figure (b) is the evolution of filament diameter, $D(t)$, and plate separation, $L(t)$, for the experiment with elongational rate of $\dot{\boldsymbol{\epsilon}}=0.03 \mathrm{~s}^{-1}$ at $120{ }^{\circ} \mathrm{C}$, stretched for $6.6 \mathrm{~s}$ and then relaxed. The diameter is kept constant by the closed loop controller in this experiment.

The first repetation based model was introduced by Doi and Edwards (1979). To explain the disagreements between mainly elongational flow, Doi and Edwards (1986) incorporated a stretch factor in this model. Based on a strain measure for an entanglement network with instantaneous chain retraction, the stress tensor is

$$
\sigma_{i j}=\lambda(t)^{2} \sigma_{D E, i j}(t) \equiv \lambda(t)^{2} \int_{-\infty}^{t} M\left(t-t^{\prime}\right) 5\left\langle\frac{E_{i n} u_{n} E_{j m} u_{m}}{|\mathbf{E} \cdot \mathbf{u}|^{2}}\right\rangle d t^{\prime}
$$

where the stretch of the tube segments, $\lambda(t)$, is an explicit function of the present time $t$. We have used the strain tensor introduced in the original work by Doi and Edwards (1979) that used the independent alignment approximation. The memory function $M(t$ $\left.-t^{\prime}\right)$ is related to the relaxation modulus as $M\left(t-t^{\prime}\right)=d G\left(t-t^{\prime}\right) / d t^{\prime}$. The angular brackets denote an average over a unit sphere $\langle\ldots\rangle=1 /(4 \pi) \int_{|\mathbf{u}|=1} \ldots d \mathbf{u}$ where a tube segment of unit length and orientation is given by the unit vector $\mathbf{u}$. The vector $\mathbf{u}$ in the stress free state is deformed into $\mathbf{E} \cdot \mathbf{u}$ in the current state. Here the components of the macroscopic displacement gradient tensor is given by $E_{i j}\left(\boldsymbol{x}, t, t^{\prime}\right)=\partial x_{i} / \partial x_{j}^{\prime}, i=1,2,3$; and $j=1,2,3$; $\left(x_{1}^{\prime}, x_{2}^{\prime}, x_{3}^{\prime}\right)$ are the coordinates of a given particle in the stress free reference state (time $\left.t^{\prime}\right)$, displaced to coordinates $\left(x_{1}, x_{2}, x_{3}\right)$ in the current state (time $t$ ).

This equation needs a stretch evolution equation, and an immense variety of concepts have been published: Doi (1980), Marrucci and Grizzuti (1988), Pearson et al. (1989), McLeish and Larson (1998), Mead et al. (1998), etc. None of these models have effectively been able to predict the extensional flow behavior of monodisperse polymer melts, although the stretch factor in the Doi-Edwards model is in principle expected to be capable of doing this.

Wagner et al. (2005) recently adopted the interchain pressure concept introduced by Marrucci and Ianniruberto (2004) into an integral molecular stress function (MSF) formulation [Wagner and Schaeffer (1992)]: 


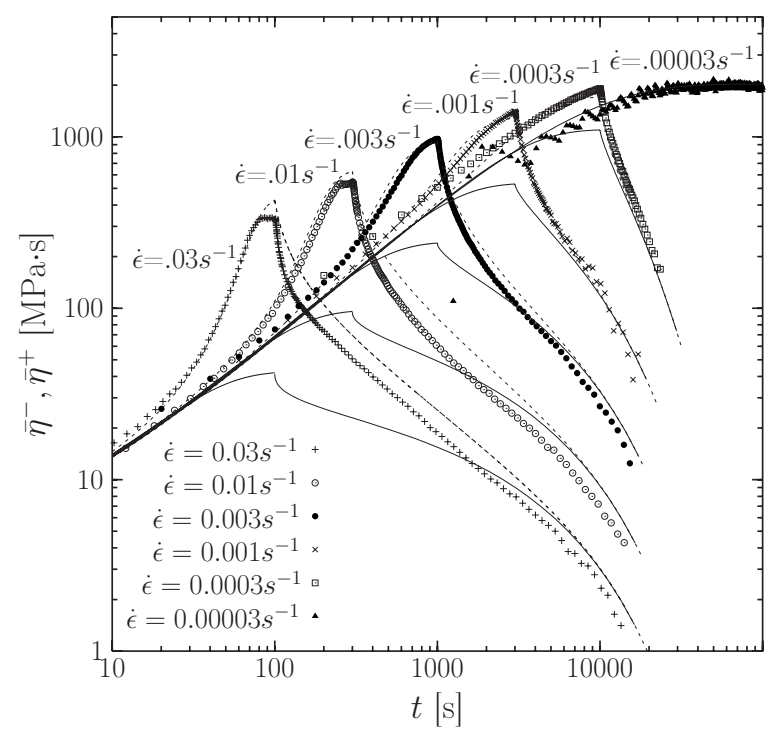

FIG. 6. The measured corrected startup and relaxation viscosity performed at rates of $\dot{\epsilon}$

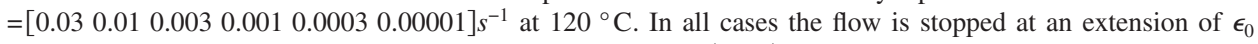
$=3$ and allowed to relax for $4 \mathrm{~h}, 14400 \mathrm{~s}$. The solid lines (-) are the Doi-Edwards predictions of the transient elongational viscosity, Eq. (8) with $\lambda(t)=1$. The dashed lines (- - -) are the MSF model prediction from Eq. (10) with a tube diameter relaxation time of $\tau_{W}=7000 \mathrm{~s}$.

$$
\sigma_{i j}^{\mathrm{MSF}}=\int_{-\infty}^{t} M\left(t-t^{\prime}\right) f\left(t, t^{\prime}\right)^{2} 5\left\langle\frac{E_{i n} u_{n} E_{j m} u_{m}}{|\mathbf{E} \cdot \mathbf{u}|^{2}}\right\rangle d t^{\prime}
$$

where

$$
\frac{\partial}{\partial t} f\left(t, t^{\prime}\right)=f\left(t, t^{\prime}\right)\left[\frac{\partial}{\partial t}\langle\ln |\mathbf{E} \cdot \mathbf{u}|\rangle-\frac{1}{\tau_{W}} f\left(t, t^{\prime}\right)\left(f\left(t, t^{\prime}\right)^{3}-1\right)\right] .
$$

$\tau_{w}$ is called the tube diameter relaxation time. The Doi-Edwards model operates with an averaged orientation factor and stretch, whereas the molecular stress function formulation considers a stretch for all deformed segments. The MSF model from Eq. (9) and (10) agrees well with measured startup and steady uniaxial elongational flow of monodisperse polystyrenes with molar masses in the range of 50-390 kg/mol [Wagner et al. (2005)].

\section{ELONGATIONAL MEASUREMENTS}

Figure 6 shows the measured corrected transient elongational viscosity, $\bar{\eta}_{\text {corr }}^{+}(t)$ followed by the viscosity $\bar{\eta}^{-}(t)$ during the stress relaxation for the NMMD polystyrene with predictions from LVE. The elongational viscosity measurements were performed at $120{ }^{\circ} \mathrm{C}$ except for the smallest elongational rates. That was measured at $150{ }^{\circ} \mathrm{C}$ and then subsequent time-temperature superposition shifted to $120^{\circ} \mathrm{C}$. The LVE predictions were based on the parameters in Table I. In both plots there is agreement between measurements and LVE predictions up to a certain level of strain. It is seen that the transient elongational viscosity rises above LVE at intermediate strains.

From Wagner et al. (2005) we have obtained a value of the $\tau_{W}=7000 \mathrm{~s}$ at $120{ }^{\circ} \mathrm{C}$ for the tube diameter relaxation time, so that $\tau_{W} / \tau_{a}=0.9$. It appears that the startup and steady elongational data in Fig. 6 are well described by the model. 


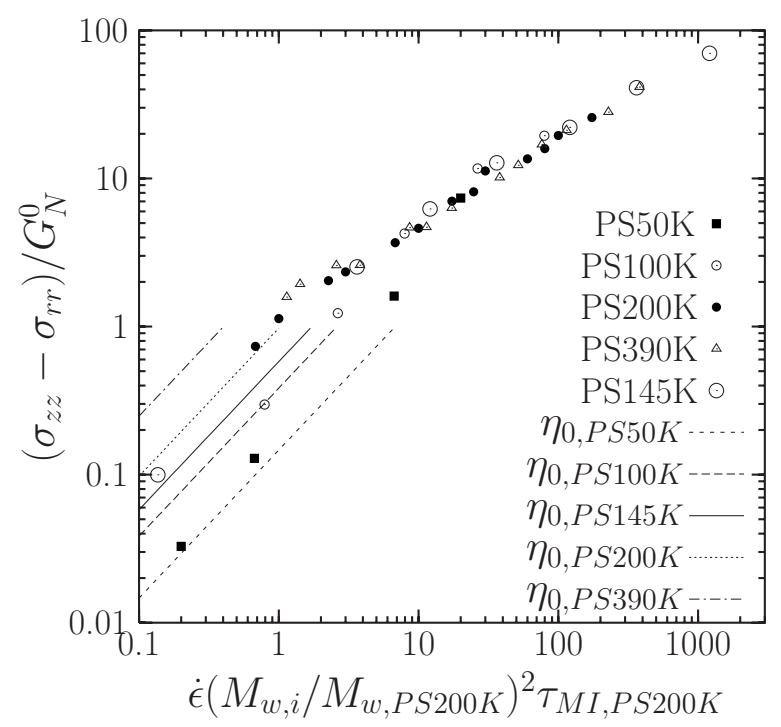

FIG. 7. The steady stress divided with the plateau modulus against the Marrucci-Deborah number $\epsilon \tau_{\mathrm{MI}}$ for PS50 K, PS100 K, PS145 K, PS200 K and PS390 K at $120^{\circ} \mathrm{C}$. The value of $\tau_{\mathrm{MI}, \mathrm{PS} 200 \mathrm{~K}}$ is $43.5 \mathrm{~s}$ at $120^{\circ} \mathrm{C} . \eta_{0}$ is the zero-shear viscosity.

To appreciate the scaling of the steady elongational viscosity with molar mass we plot the measured steady state elongational stresses as $\left(\sigma_{z z}-\sigma_{r r}\right) / G_{N}^{0}$ versus $\dot{\epsilon} \tau_{\mathrm{MI}} . \tau_{\mathrm{MI}}$ is the tube diameter relaxation time, which is defined by Marrucci and Ianniruberto (2004) (MI). Marrucci and Ianniruberto report $\tau_{\mathrm{MI}}$ at $130{ }^{\circ} \mathrm{C}$ for PS200 to be $\tau_{\mathrm{MI}}=1 \cdot 10^{3} \mathrm{~s}$, i.e., $\tau_{\mathrm{MI}}=23 \cdot 10^{3} \mathrm{~s}$ at $120^{\circ} \mathrm{C}$, and the scaling is $\tau_{\mathrm{MI}} \sim M_{w}^{2}$. This gives a value of $14 \cdot 10^{3}$ for $M_{W}=145 \mathrm{~kg} / \mathrm{mol}$ which is used to plot the steady data in Fig. 7 . We have included the steady state values measured by Bach et al. (2003a) and Nielsen et al. (2006a). It is seen that the scaled values for all measurements collapse onto one single power law behavior for $\sigma_{z z}-\sigma_{r r}>G_{n}^{0}$. For $\sigma_{z z}-\sigma_{r r}<G_{n}^{0}$ the data lie on the five lines indicating the zero shear viscosity.

\section{STRESS RELAXATION}

The stretch factor $f\left(t, t^{\prime}\right)$ in the MSF model, Eq. (9), depends both on the time of creation $\left(t^{\prime}\right)$ of segments and the present time $(t)$. Consequently, at any time, $t$, it is not possible to assign a single value to the polymer stretch. If, however, one uses the constitutive integral form from Eq. (8), where the stretch factor $\lambda^{2}$ is placed in front of the integral, it is possible to compute a single stretch factor that applies for every startup and relaxation experiment. It is of interest to compare the experimentally observed stresses during the relaxation with those obtained from the Doi-Edwards approach in this alternative representation. Following that method the stretch factor can be obtained experimentally from:

$$
\lambda(t)^{2}=\frac{\sigma_{z z}-\sigma_{r r}}{\sigma_{D E, z z}-\sigma_{D E, r r}},
$$

where $\sigma_{z z}-\sigma_{r r}$ is the experimentally measured stress from Eq. (5) and $\sigma_{\mathrm{DE}}(t)$ is given in Eq. (8). The experimentally determined stretch factor is shown as a function of the nondimensional time in Fig. 8, with the strain based on a the strain measure for an 


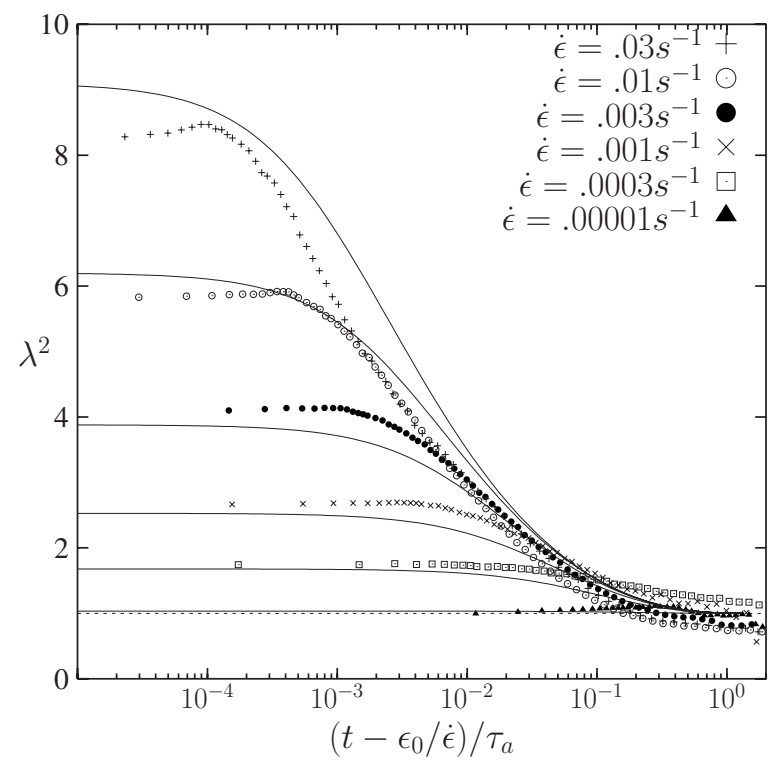

FIG. 8. The stretch, $\lambda^{2}$, calculated using the measured relaxation viscosity at $120{ }^{\circ} \mathrm{C}$ in Fig. 6 , as a function of the time, from the start of the stress relaxation. The stretch is found using Eq. (11) based on the independent alignment approximation, Eq. (8). The solid lines are the stretch calculated using Eq. (13), with a tube diameter relaxation time $\left(\tau_{\mathrm{MI}}\right)$ and stretch of the fully extended molecule $\left(\lambda_{\max }\right)$ of $\left(\tau_{\mathrm{MI}}, \lambda_{\max }^{2}\right)=(5500 \mathrm{~s}, 22)$.

entanglement network with the independent alignment approximation $\left(\lambda^{2}\right)$, respectively. We note that at long times the experimental stretch values, $\lambda^{2}$, approach unity, but the experimental accuracy seems to decrease somewhat with time. Furthermore, the curves from several different stretch rates seem to converge on a common envelope.

Pearson et al. (1989) suggested the stretch factor follow a first order differential equation in the present time $t$. For completeness we give the analytical solution of the stretch during stress relaxation, by Pearson et al. (1989); $\lambda(t)=1$ $+\left(\lambda\left(\epsilon_{0} / \dot{\epsilon}\right)-1\right) \exp \left(\frac{\epsilon_{0} / \dot{\epsilon}-t}{C}\right) . C$ is a material dependent time constant and $\lambda\left(\epsilon_{0} / \dot{\epsilon}\right)$ is the value of the stretch where the flow stops. This first order model will yield separate curves for $\lambda^{2}(t)$ that do not converge except for $\lambda \rightarrow 1$ in the limit $t \rightarrow \infty$. By contrast the observed $\lambda(t)$ curves seem to converge at a common envelope before $\lambda \rightarrow 1$.

Adopting the interchain pressure concept within the Doi-Edwards model we may gain more insight into the stretch mechanism. Marrucci and Ianniruberto (2004) derived an equation for the tube diameter, $a$, in a uniaxial elongational flow, based on the interchain pressure concept. The tube diameter $a$ follows the expression:

$$
\frac{\partial a}{\partial t}=-\dot{\epsilon} a+\frac{a_{0}}{\tau_{\mathrm{MI}}}\left(c^{2} \frac{a_{0}^{3}}{a^{3}}-1\right) .
$$

where $\tau_{\mathrm{MI}}$ is a tube diameter relaxation time, $a_{0}$ the initial diameter and $c$ a nonlinear spring coefficient represented by a relative Pade approximation [Cohen (1991)]. Marrucci and Ianniruberto (2004) did not include finite extensibility and therefore assumed $c=1$. Rolón-Garrido et al. (2006) later replaced the stretch factor with the inverse tube diameter, $\lambda=a_{0} / a$, and introduced finite extensibility in Eq. (12), obtaining an expression for the stretch: 


$$
\frac{\partial \lambda}{\partial t}=\dot{\epsilon} \lambda-\frac{\lambda^{2}}{\tau_{\mathrm{MI}}}\left(c(\lambda)^{2} \lambda^{3}-1\right) .
$$

From this equation we see that $\tau_{\mathrm{MI}}$ may be interpreted also as a stretch relaxation time. The relative Pade inverse Langevin function [Ye and Sridhar (2005)] is

$$
c(\lambda)=\frac{\left(3-\lambda^{2} / \lambda_{\max }^{2}\right)\left(1-1 / \lambda_{\max }^{2}\right)}{\left(3-1 / \lambda_{\max }^{2}\right)\left(1-\lambda / \lambda_{\max }^{2}\right)},
$$

where $\lambda_{\max }$ is the stretch of the fully extended molecule. For polystyrene we use $\lambda_{\max }$ $=4.7$ [Fang et al. (2000)].

In stress relaxation $\dot{\boldsymbol{\epsilon}}=0$. The corresponding solution to Eq. (13) is shown in Fig. 8 starting from the steady state values of $\lambda$. We have used the constant $\tau_{\mathrm{MI}}=5500 \mathrm{~s}$. We note in closing that this value is somewhat smaller than the value obtained by the molecular weight scaling argument. While the model shows the correct relaxation it does not display the convergence of the individual curves to an envelope.

\section{CONCLUSION}

A filament stretching apparatus was used to measure the uniaxial elongational viscosity followed by stress relaxation using a $145 \mathrm{~kg} / \mathrm{mol}$ narrow molar mass distribution polystyrene melt. The closed loop control in the filament stretching apparatus was extended to handle the stress relaxation. Here the end plates in the filament stretching rheometer were controlled to ensure the no flow condition, corresponding to no change in the midfilament diameter. The main conclusions of this work are:

- The experimentally determined stretch factors all converge to unity for large time. This supports the concept of factorization of stress into a tensorial contribution determined by repetation dynamics, modified by a scalar factor due to stretch. In the ultimate stress relaxation, the stress is dominated by the contribution from the repetation dynamics.

- The experimentally determined stress relaxation time is of the same order of magnitude as the repetation time. This may seem at variance with our observation that the ultimate stress relaxation is described by repetation. However, there is no conflict since stretch becomes a higher order effect in the limit as $\lambda \rightarrow \infty$ in Eq. (7).

- Currently available theories do not reproduce the complex and highly nonlinear behavior of the experimentally determined stretch factors.

\section{ACKNOWLEDGMENTS}

The authors gratefully acknowledge the financial support of the Graduate School of Polymer Science from the Danish Research Training Council, and the Danish Technical Research Council to the Danish Polymer Center.

\section{References}

Anna, S. L., C. B. Rogers, and G. H. McKinley, "On controlling the kinematics of a filament stretching rheometer using a real-time active control mechanism," J. Non-Newtonian Fluid Mech. 87, 307-335 (1999).

Bach, A., K. Almdal, H. K. Rasmussen, and O. Hassager, "Elongational viscosity of narrow molar mass distribution polystyrene," Macromolecules 36, 5174-5179 (2003a). 
Bach, A., H. K. Rasmussen, and O. Hassager, "Extensional viscosity for polymer melts measured in the filament stretching rheometer," J. Rheol. 47, 429-441 (2003b).

Baumgaertel, M., A. Schausberger, and H. H. Winter, "The relaxation of polymers with linear flexible chains of uniform length," Rheol. Acta 29, 400-408 (1990).

Bhattacharjee, P. K., D. A. Nguyen, G. H. McKinley, and T. Sridhar, "Extensional stress growth and stress relaxation in entangled polymer solutions," J. Rheol. 47, 269-290 (2003).

Bird, R. B., R. C. Armstrong, and O. Hassager, Dynamics of Polymeric Liquids, Vol. I, Fluid Mechanics, (Wiley, New York, 1987).

Cohen, A., "A Pade approximant to the inverse Langevin function," Rheol. Acta 30, 270-273 (1991).

Doi, M., "Constitutive equation derived from the model of Doi and Edwards for concentrated polymer solutions and polymer melts,” J. Polym. Sci., Polym. Phys. Ed. 18, 2055-2067 (1980).

Doi, M., and S. F. Edwards, "Dynamics of concentrated polymer systems. VI. Rheological properties," J. Chem. Soc., Faraday Trans. 1 75, 38-54 (1979).

Doi, M., and S. F. Edwards, The Theory of Polymer Dynamics (Clarendon, Oxford, 1986).

Doyle, P. S., E. S. G. Shaqfeh, G. H. McKinley, and S. H. Spiegelberg, "Relaxation of dilute polymer solutions following extensional flow,” J. Non-Newtonian Fluid Mech. 76 79-110 (1998).

Fang, J., M. Kröger, and H. C. Öttinger, "A thermodynamically admissible repetation model for fast flows of entangled polymers. II. Model predictions for shear and extensional flows," J. Rheol. 44, 1293-1317 (2000).

Ianniruberto, G., and G. Marrucci, "A simple constitutive equation for entangled polymers with chain stretch," J. Rheol. 45, 1305-1318 (2001).

Jackson, J. K., and H. H. Winter, "Entanglement and Flow Behavior of Bidisperse Blends of Polystyrene and Polybutadiene," Macromolecules 28, 3146-3155 (1995).

Kolte, M. I., H. K. Rasmussen, and O. Hassager, "Transient filament stretching rheometer II: Numerical simulation," Rheol. Acta 36, 285-302 (1997).

Likhtman, A., "Single-chain slip-link model of entangled polymers: Simultaneous description of neutron spinecho, Rheology, and Diffusion," Macromolecules 38, 6128-6139 (2005).

Luap, C., C. Müller, T. Schweizer, and D. C. Venerus, "Simultaneous stress and birefringence measurements during uniaxial elongation of polystyrene melts with narrow molecular weight distribution," Rheol. Acta 45, 83-91 (2005).

Marrucci, G., and N. Grizzuti, "Fast flows of concentrated polymers-Predictions of the tube model on chain stretching,” Gazz. Chim. Ital. 118, 179-185 (1988).

Marrucci, G., and G. Ianniruberto, "Interchain pressure effect in extensional flows of entangled polymer melts," Macromolecules 37, 3934-3942 (2004).

McKinley, G. H., and T. Sridhar, "Filament-stretching rheometry of complex fluids," Annu. Rev. Fluid Mech. 32, 375-416 (2002).

McLeish, T. C. B., and R. G. Larson, "Molecular constitutive equations for a class of branched polymers: The pom-pom polymer," J. Rheol. 42, 81-110 (1998).

Mead, D. W., R. G. Larson, and M. Doi, "A molecular theory for fast flows of entangled polymers," Macromolecules 31, 7895-7914 (1998).

Ndoni, S., C. M. Papadakis, F. S. Bates, and K. Almdal, "Laboratory-scale setup for anionic polymerization under inert atmosphere,” Rev. Sci. Instrum. 66, 1090-1095 (1995).

Nielsen, J. K., H. K. Rasmussen, O. Hassager, and G. H. McKinley, "Elongational viscosity of monodisperse and bidisperse polystyrene melts," J. Rheol. 50, 453-476 (2006a).

Nielsen, J. K., H. K. Rasmussen, M. Denberg, K. Almdal, and O. Hassager, "Nonlinear branch-point dynamics of multiarm polystyrene," Macromolecules 39, 8844-8853 (2006b).

Nieuwkoop, J. van, and M. M. O. M. von Czernicki, "Elongation and subsequent relaxation measurements on dilute polyisobutylene," J. Non-Newtonian Fluid Mech. 67, 105-123 (1996).

Orr, N. V., and T. Sridhar, "Stress relaxation in uniaxial extension," J. Non-Newtonian Fluid Mech. 67, 77-103 (1996).

Pearson, D. S., A. D. Kiss, L. J. Fetters, and M. Doi, "Flow-induced birefringence of concentrated polyisoprene solutions," J. Rheol. 33, 517-535 (1989). 
Rasmussen, H. K., P. Laillé, and K. Yu, "Large amplitude oscillatory elongation flow,” Rheol. Acta 47, 97-103 (2008).

Rasmussen, H. K., J. H. Christensen, and S. J. Gottsche, "Inflation of polymer melts into elliptic and circular cylinders," J. Non-Cryst. Solids 93, 245-263 (2000).

Rodd, L. E., T. P. Scott, J. J. Cooper-White, and G. H. McKinley, "Capillary breakup rheometry of lowviscosity elastic fluids," Appl. Rheol. 15, 12-27 (2005).

Rolón-Garrido, V. H., M. H. Wagner, C. Luap, and T. Schweizer, "Modeling non-Gaussian extensibility effects in elongation of nearly monodisperse polystyrene melts," J. Rheol. 50, 327-340 (2006).

Schausberger, A., and G. Schindlauer, "Linear elastico-viscous properties of molten standard polystyrenes," J. Rheol. 24, 220-227 (1985).

Schieber, J., J. Neergaard, and S. Gupta, "A full-chain, temporary network model with sliplinks, chain-length fluctuations, chain connectivity and chain stretching," J. Rheol. 47, 213-233 (2003).

Sentmanat, M., B. N. Wang, and G. H. McKinley, "Measuring the transient extensional rheology of polyethylene melts using the SER universal testing platform," J. Rheol. 49, 585-606 (2005).

Shaqfeh, E. S. G., G. H. McKinley, N. Woo, D. A. Nguyen, and T. Sridhar, "On the polymer entropic force singularity and its relation to extensional stress relaxation and filament recoil," J. Rheol. 48, 209-221 (2004).

Spiegelberg, S. H., and G. H. McKinley, "Stress relaxation and elastic decohesion of viscoelastic polymer solutions in extensional flow," J. Non-Newtonian Fluid Mech. 67, 49-76 (1996a).

Spiegelberg, S. H., and G. H. McKinley, "The role of end-effects on measurements of extensional viscosity in filament stretching rheometers," J. Non-Newtonian Fluid Mech. 64, 229-267 (1996b).

Sridhar, T., V. Tirtaadmadja, D. A. Nguyen, and R. K. Gupta, "Measurement the extensional viscosity of polymer-solutions," J. Non-Newtonian Fluid Mech. 40, 271-280 (1991) and "Chain connectivity and chain stretching," J. Rheol. 47, 213-233 (2003).

Szabo, P., and G. H. McKinley, "Filament stretching rheometer: Inertia compensation revisited," Rheol. Acta 42, 269-271 (2003).

Szabo, P., "Transient filament stretching rheometer part I: Force Balance analysis," Rheol. Acta 36, 277-284 (1997).

Wagner, M. H., and J. Schaeffer, "Nonlinear strain measures for general biaxial extension of polymer melts," J. Rheol. 36, 1-26 (1992).

Wagner, M. H., S. Kheirandish, and O. Hassager, "Quantitative prediction of the transient and steady-state elongatiuonal viscosity of nearly monodisperse polystyrene melts," J. Rheol. 49, 1317-1327 (2005).

Wagner, M. H., V. H. Rolón-Garrido, J. K. Nielsen, H. K. Rasmussen, and O. Hassager, "A constitutive analysis of transient and steady-state elongational viscosities of bidisperse polystyrene blends," J. Rheol. 52, 67-86 (2008).

Wang, Y., P. Boukany, S.-Q. Wang, and X. Wang, "Elastic breakup in uniaxial extension of entangled polymer melts," Phys. Rev. Lett. 99, 237801 (2007).

Ye, X., and T. Sridhar, "Effects of the polydispersity on rheological properties of entangled polystyrene solutions," Macromolecules 38, 3442-3449 (2005). 